\title{
Improving Compressed Iris Recognition Accuracy Using JPEG2000 RoI Coding
}

\author{
J. Hämmerle-Uhl, C. Prähauser, T. Starzacher, and A. Uhl \\ Department of Computer Sciences, Salzburg University, Austria \\ uhl@cosy.sbg.ac.at
}

\begin{abstract}
The impact of using JPEG2000 region of interest coding on the matching accuracy of iris recognition systems is investigated. In particular, we compare the matching scores as obtained by a concrete recognition system when using JPEG2000 compression of rectilinear iris images with and without region of interest coding enabled. The region of interest is restricted to the iris texture area plus the pupil region. It turns out that average matching scores can be improved and that the number of false negative matches is significantly decreased using region of interest coding as compared to plain JPEG2000 compression.
\end{abstract}

\section{Introduction}

Iris recognition is claimed to be the most secure biometric modality exhibiting practically $0 \% \mathrm{FAR}$ and low FRR. An interesting fact is that the iris recognition market is strongly dominated by Iridian Inc. based technology which is based on algorithms of J. Daugman [1. Certain application scenarios may require compression technology being applied to sample data: In distributed biometric systems, the data acquisition stage is often dislocated from the feature extraction and matching stage (this is true for the enrolment phase as well as for authentication). In such environments the sensor data have to be transferred via a network link to the respective location, often over wireless channels with low bandwidth and high latency. Therefore, a minimisation of the amount of data to be transferred is highly desirable, which is achieved by compressing the data before transmission.

Having found that compression of the raw sensor data can be advantageous in certain applications, we have to identify techniques suited to accomplish this task in an optimal manner. In order to maximise the benefit in terms of data reduction, lossy compression techniques have to be applied. However, the distortions introduced by compression artifacts may interfere with subsequent feature extraction and may degrade the matching results. In particular, FRR or FNMR will increase (since features of the data of legitimate users are extracted less accurately from compressed data) which in turn affects user convenience and general acceptance of the biometric system. In extreme cases, even FAR or FMR might be affected. Therefore, careful optimisation of compression techniques employed is highly desirable. 
The certainly most relevant standard for compressing iris image data is the recent ISO/IEC 19794-6 standard on Biometric Data Interchange Formats which allows iris image data to be stored in lossy manner in the JPEG and JPEG2000 formats. Two types of iris image data are considered: rectilinear images (i.e. images of the entire eye) and polar images (which are basically the result of iris detection and segmentation), the latter much smaller in terms of storage requirement (e.g. $2 \mathrm{kB}$ vs. $25-30 \mathrm{kB}$ for rectilinear images). It is important to note that with this standardisation it might become more attractive for companies to investigate alternatives to Iridian products due to the available common data format iris recognition systems can rely on.

Only recently, first results and techniques are available on iris image compression and its impact on recognition performance. Ives et al. 4, 3] apply JPEG2000 up to a compression rate of 20 to rectilinear image data (the CASIA database and a proprietary image collection is used) and investigate the effects on FAR and FRR of a 1-D version of the Daugman algorithm (the same system which is used in this study). Rakshit and Monro 9] again use JPEG2000 to compress polar iris images up to a compression rate of 80 and study the impact on verification accuracy of three iris recognition systems (including the Daugman algorithm, the CASIA database is used). Daugman and Downing 2 apply JPEG and JPEG2000 to rectilinear image data (the NIST ICE database is used) and remove image background (i.e. parts of the image not being part of the eye like eye-lids are replaced by constant average gray) before compression is applied. A more compact way of representing the Daugman IrisCode is discussed in [1], however, these results refer to template compression and are only valid for the techniques related to Iridian products.

Five general purpose compression algorithms (including JPEG and JPEG2000) are compared in [6, 5] with respect to their impact on iris recognition accuracy of three different recognition schemes (the CASIA and UBIRIS databases have been used). In accordance to 2 significantly superior compression performance of JPEG2000 over JPEG especially for low bitrates have been found. So far, compression algorithms including JPEG2000 have been applied to iris imagery with their respective standard settings.

In the subsequent experimental study we apply JPEG2000 to rectangular iris data. JPEG2000 region of interest (RoI) coding is applied to the iris texture in order to preserve more textural detail in this area of the images and the corresponding iris recognition results are compared to those obtained by applying plain JPEG2000 compression.

Section 2 introduces the proposed JPEG2000 RoI iris coding framework. Section 3 is the experimental part of this work where we first describe the iris recognition system used to evaluate the JPEG2000 based approach, and the data these algorithms are applied to. Subsequently, we present and discuss our experimental results where we compare iris recognition results using JPEG2000 compression with and without RoI coding enabled. Section 4 concludes the paper. 


\section{JPEG2000 Region of Interest Coding of Iris Images}

The rational for our approach is as follows: Since iris recognition accuracy relies only on the iris texture data, we propose a selective compression approach for iris image data. After detection of the quasi-circular iris region in a rectangular iris image, we pass the information about the identified circular Region of Interest (RoI) to the JPEG2000 encoder which allocates higher quality to this RoI as compared to the rest of the image employing its RoI compression capability. Using this strategy, better iris recognition accuracy is expected as in the case of standard JPEG2000 compression, where the bitrate is distributed evenly among the different regions in the image.

\subsection{Iris Detection}

Our iris detection approach employs a sequence of standard image processing operations:

1. Histogram equalisation: low contrast images are enhanced to give better results in the subsequent processing stages.

2. Canny edge detection.

3. Canny edges are fed into a Hough transform for circle detection. The circle with the largest radius in a specific range is our detected outer iris boundary.

After the circle detection was successful, we select the detected circle in the image as Region of Interest which is passed to the JPEG2000 encoder.

\subsection{JPEG2000 RoI Coding}

The JPEG2000 10] image coding standard uses the wavelet transform as energy compaction method. JPEG2000 may be operated in lossy and lossless mode (using a reversible integer transform in the latter case) and also the wavelet decomposition depth may be defined. The major difference between previously proposed zerotree wavelet-based image compression algorithms such as EZW or SPIHT is that JPEG2000 operates on independent, non-overlapping blocks of transform coefficients ("codeblocks"). After the wavelet transform, the coefficients are quantized and encoded on a codeblock basis using the EBCOT scheme, which renders distortion scalability possible. Thereby the coefficients are grouped into codeblocks and these are encoded bitplane by bitplane, each with three coding passes (except the first bitplane). The codeblock size can be chosen arbitrarily with certain restrictions.

main packet packet ... packet packet

header header data ... header data

Fig. 1. JPEG2000 bitstream structure 
The final JPEG2000 bitstream (see Fig. 1) is organised as follows: The main header is followed by packets of data (packet bodies) each of which is preceded by a packet header. A packet body contains CCPs (codeblock contribution to packet) of codeblocks that belong to the same image resolution (wavelet decomposition level) and layer (which roughly stand for successive quality levels). Depending on the arrangement of the packets, different progression orders may be specified. Resolution and layer progression order are the most important progression orders for grayscale images.

In our experiments, we have used the JAVA reference implementation JJ200¿1 which also supports RoI coding which is described in the following.

RoI coding is a feature that enables a non-uniform distribution of the image quality. Parts of the image can be defined as RoI, which are usually areas that contain objects which are of particular interest to the application. An image may contain multiple disjoint RoIs. Although it is possible to define RoIs with arbitrary shapes, the more common cases are rectangles, circles or other simple geometric forms. The image data where a lower (relative) quality is acceptable is called the Background (BG). The BG is comprised of all image parts that do not belong to a RoI. RoIs are discussed in Annex H of the JPEG2000 standard [10].

The technical solution to achieve RoI coding is described next. RoIs are often described by simple geometrical shapes. In the JPEG2000 encoding process, the first step is then to derive from this geometric objects in the spatial domain appropriate areas in the wavelet domain. These areas, called RoI Masks, contain the wavelet coefficients that belong to RoIs. The method used for priorizing RoI coefficients during the encoding process is called MaxShift. MaxShift exploits a property of the EBCOT coding algorithm [10]: The entropy coding for a code block always starts at the most significant bit plane of the coefficients. Thus, information contained in higher bit planes is encoded before the information in lower bit planes. MaxShift simply scales the coefficients belonging to the BG in such a way, that the highest bit plane to which any BG coefficient contributes is lower than the lowest bit plane to which any RoI coefficient contributes.

The exact process at the encoder is described as follows:

1. The encoder determines a scaling value $s$ with the following property:

$$
\begin{array}{r}
\text { Let } k_{\text {RoImin }}=2^{s} \\
\forall k_{B G} \in B G: k_{\text {RoImin }}>k_{B G}
\end{array}
$$

2. All coefficients of the Background are scaled down (shifted right) by the scaling value $s$.

3. The scaling value $s$ is inserted in the resulting JPEG2000 code stream.

At the decoder side, the process is reversed:

1. The decoder extracts the scaling value $s$ from the bit stream.

2. Based on the scaling value the decoder can determine if a coefficient $k$ belongs to a Region of Interest:

${ }^{1}$ http://jj2000.epfl.ch/ 


$$
\begin{array}{r}
k \geq k_{\text {RoImin }} \Longrightarrow k \in R o I \\
k<k_{\text {RoImin }} \Longrightarrow k \notin R o I \Longrightarrow k \in B G
\end{array}
$$

3. All coefficients belonging to the BG are now scaled up to their original value using the scaling value $s$.

\section{Experimental Study}

\subsection{Iris Recognition System}

The employed iris recognition system is Libor Masek's Matlab implementation 2 of a 1-D version of the Daugman iris recognition algorithm. First, this algorithm segments the eye image into the iris and the remainder of the image. After extracting the features of the iris (which are strongly quantized phase responses of complex 1-D Gabor filters in this case), considering translation, rotations and disturbed regions in the iris (a noise mask is generated), the algorithm outputs the similarity score by giving the Hamming distance between two extracted templates. The range of the Hamming distance reaches from zero (ideal matching of two iris images of the same person) to 0.5 (ideal mismatch between two iris images of different persons).

\subsection{Sample Data}

We use images from two databases for our tests (see table 1). The first images are taken from the Chinese Academy of Sciences V Institute of Automation (CASIA $\left.{ }^{3}\right)$. Each image from the CASIA database is stored as a 8-bit (indexed) gray-scale with a resolution of 320 by 280 pixels. The pictures are taken with a camera using near-infrared light for illumination. This avoids reflections (especially in the dark black pupil area) and may yield a finer structure of the iris. Note that the fact that the pupil area has been manipulated in these data [7] is not relevant in our context since templates for matching are extracted from the polar iris patch only. Moreover, these data come in uncompressed format which is important for the rating of subsequent compression.

The second database we use is UBIRIS [8]. The images are taken with a "normal" (non-infrared) camera and have a size of 200 by 150 pixels in 8-bit gray-scale format (JPEG compressed).

Figure 2 shows an example of a non-RoI JPEG2000 compressed (compression rate 96) iris image of one person (CASIA data), which was used in our calculations together with the extracted iris template data and the noise masks (template and noise mask have been scaled in y-direction by a factor of 4 for proper display).

The differences in the templates are more significant as those in the noise masks which turn out to be very similar. Overall also iris detection is very

\footnotetext{
${ }^{2}$ http://www.csse.uwa.edu.au/ pk/studentprojects/libor/sourcecode.html

3 http://www.sinobiometrics.com
} 
Table 1. Eye-Image Databases used for Tests

\begin{tabular}{|l|c|c|}
\hline & CASIA & UBIRIS \\
\hline Width & 320 & 200 \\
\hline Height & 280 & 150 \\
\hline Pixels & 89600 & 30000 \\
\hline Format & $\begin{array}{c}\text { 8-bit gray-scale } \\
\text { BMP }\end{array}$ & $\begin{array}{c}\text { 8-bit gray-scale } \\
\text { JPEG }\end{array}$ \\
\hline Number of Image Sets & 40 & 20 \\
\hline Number of Images per Image Set & 3 & 4 \\
\hline
\end{tabular}
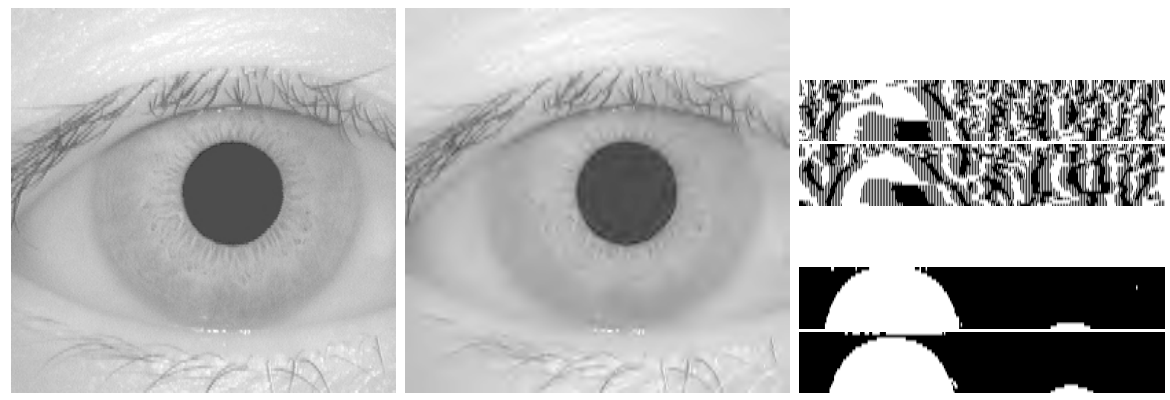

Fig. 2. Comparison of uncompressed/compressed iris image and the corresponding iris templates and noise masks

robust to compression - all matching results shown in the following section have been generated without the software failing to detect the iris.

Compression can be used in various stages of the matching process. Either the stored reference data may be in compressed form, or the sample data acquired for verification may be compressed (e.g. for efficient transfer), or both. In this study, we consider the case of one image out of two involved in matching is employed in compressed manner.

\subsection{Experimental Results}

For the CASIA images, on average $38 \%$ of the image area belongs to the iris. For UBIRIS, the average size of the iris is $20 \%$. We performed the tests for JPEG2000 coding with rates of $0.3,0.2,0.1$ and 0.08 bits per pixel (bpp) on the CASIA image sets and coding rates of $0.8,0.7,0.6$ and $0.5 \mathrm{bpp}$ for the UBIRIS image sets. Note that in the case of UBIRIS images, we apply a re-compression to JPEGcompressed imagery where results are different as compared to compressing raw image data [5]. We measure Hamming distance (HD) and plot on the y-axis $1-H D$ as the matching score.

Figure 3 shows the average matching results over all image sets considered, we compare the application of JPEG2000 with default settings (J2K) to the employment of the proposed RoI functionality (J2KwROI). For the CASIA image sets (Fig. 3.a), the average matching score for the uncompressed case (original) is 
slightly above 0.71 . For the four bitrates considered, we find the variant exploiting the RoI functionality being consistently superior to the default JPEG2000 case, the matching score is constantly higher by approximately 0.02 and reaches almost uncompressed performance at $0.3 \mathrm{bpp}$.

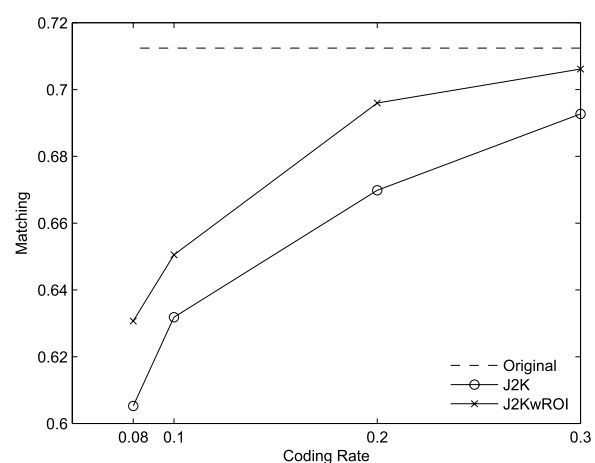

(a) CASIA

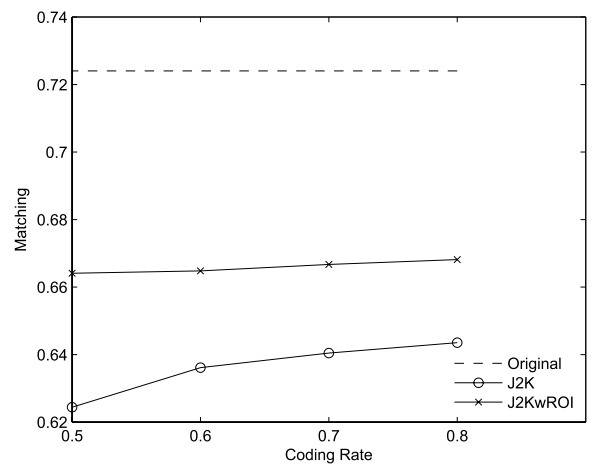

(b) UBIRIS

Fig. 3. Average matching scores over all image sets (Coding Rate in bpp)

For the UBIRIS image sets, average matching score is slightly above 0.72 in the "uncompressed" case (recall that UBIRIS images already come as JPEG files). Here, superiority of the RoI approach is even more pronounced, the observed matching score gain is in the range $0.02-0.04$ and decreases for increasing bitrate.

Having documented the gain in terms of average matching score, it is not yet clear in how far this gain will impact on actual recognition performance. In order to investigate this in more depth, we provide averaged matching score results for each image set separately. Fig. 4 shows the corresponding results for the CASIA image sets compressed at $0.2 \mathrm{bpp}$. For reference, also a hypothetical decision

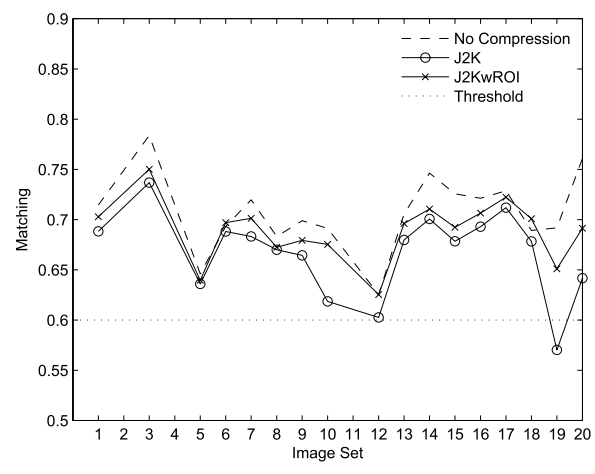

(a) Image Sets 1 to 20

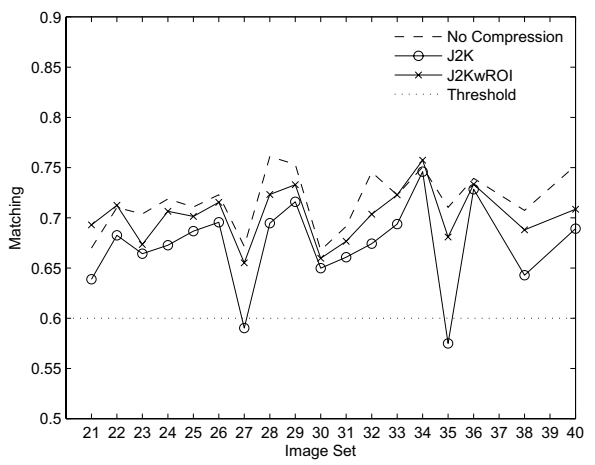

(b) Image Sets 21 to 40

Fig. 4. CASIA: Matching scores at coding rate $0.2 \mathrm{bpp}$ 
threshold value is included in the plot at matching score 0.6 (corresponding to $H D=0.4$ accordingly).

We notice, that for applying JPEG2000 with RoI functionality, matching score values stay well above 0.6 (even above 0.65 for all but sets 5 and 12), while for the standard JPEG2000 case we find values below the threshold for sets 19, 27, and 35 .

Fig. 5 shows corresponding results for the CASIA sets $1-20$ at 0.3 bpp and the UBIRIS sets at $0.5 \mathrm{bpp}$. For the CASIA sets, at the higher bitrate now no set is blow the decision threshold, while the superior performance of J2KwRoI is still visible. For the UBIRIS sets (Fig. 5. b), again the superiority of J2KwRoI is clearly visible. In addition to the better overall values, only 1 image set results in a matching score below the threshold while 3 set are affected in the default J2K case.

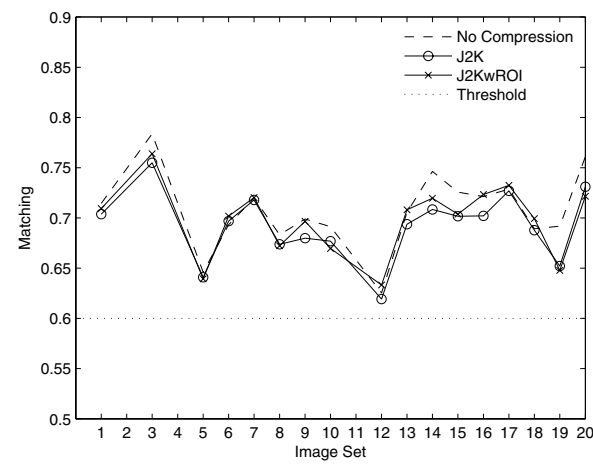

(a) CASIA Sets 1 to 20: Rate $0.3 \mathrm{bpp}$

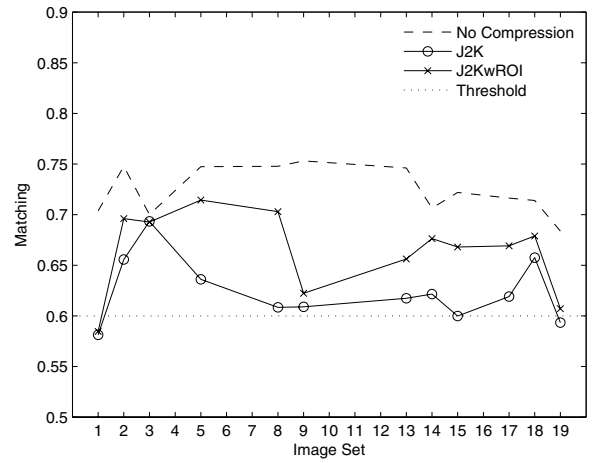

(b) UBIRIS Sets 1 to 20: Rate $0.5 \mathrm{bpp}$

Fig. 5. Matching scores at coding rates $0.3 \mathrm{bpp}$ and $0.5 \mathrm{bpp}$ for CASIA and UBIRIS

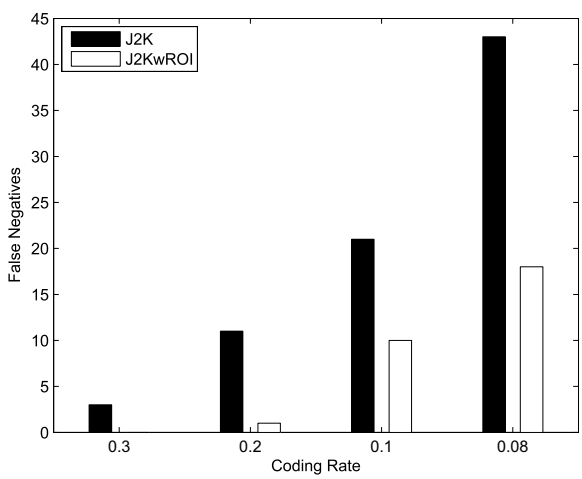

(a) CASIA

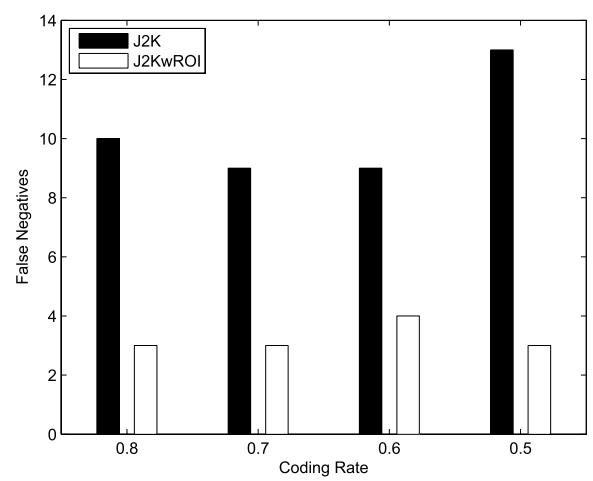

(b) UBIRIS

Fig. 6. False Negatives $\left(T_{\text {Match }}=0.6\right)$ 
In order to get entirely rid of averaging effects in the comparisons (note that HD-results of each image set are still averaged in the latter results), we provide the number of false negative matches setting the decision threshold to 0.6 (which can be used to compute the false non-match rate (FNMR)). Fig 6 shows corresponding results. For both types of images, the number of false negatives is significantly lower in case of using the RoI approach as compared to the default usage of JPEG2000.

This means, that obviously the ROC is improved by employing the proposed approach, especially in the area of improving user convenience by significantly reducing the number of false negative matches.

\section{Conclusion and Future Work}

We have investigated the impact of using JPEG2000 region of interest coding on the matching accuracy of iris recognition systems. The region of interest is restricted to the iris texture area plus the pupil region. We find that average matching scores can be improved and that the number of false negative matches is significantly decreased using region of interest coding as compared to plain JPEG2000 compression. This implies an increased user convenience at an at least equal level of security (we have not found evidence for false positives using either technique).

In a certain sense, our approach is related to [2] since both techniques allocate a lower bitrate to the image regions not containing the iris. While the latter technique entirely removes image parts not containing the iris by creating an artificial image containing only pupil, iris texture, and uniform gray pixels, our approach retains the image background with a lower quality and maintains the image integrity.

As a consequence, our approach features the following advantage over [2]: in case the iris detection process fails, our approach retains the entire iris texture (though with reduced quality in the areas which have been falsely detected as being non-iris) while using [2], the corresponding parts of the iris are cut off and replaced by constant gray pixels.

In future work we will compare both approaches with respect to their impact on recognition accuracy and will focus on sensible manners to combine both techniques.

\section{Acknowledgements}

This work has been partially supported by the Austrian Science Fund, project no. L554-N15. Most of the work described in this paper has been done in the scope of a semester project (seminar on "Visual Computing and Multimedia") in the master program on "Applied Computer Science" at the University of Salzburg. 


\section{References}

[1] Daugman, J.: How iris recognition works. IEEE Transactions on Circiuts and Systems for Video Technology 14(1), 21-30 (2004)

[2] Daugman, J., Downing, C.: Effect of severe image compression on iris recognition performance. IEEE Transactions on Information Forensics and Security 3(1), 52 $61(2008)$

[3] Ives, R.W., Broussard, R.P., Kennell, L.R., Soldan, D.L.: Effects of image compression on iris recognition system performance. Journal of Electronic Imaging 17, 011015 (2008)

[4] Ives, R.W., Bonney, B.L., Etter, D.M.: Effect of image compression on iris recognition. In: IMTC 2005 - Instrumentation and Measurement Technology Conference (May 2005)

[5] Jenisch, S., Lukesch, S., Uhl, A.: Comparison of compression algorithms' impact on iris recognition accuracy II: revisiting JPEG. In: Proceedings of SPIE, Security, Forensics, Steganography, and Watermarking of Multimedia Contents X, San Jose, CA, USA, vol. 6819, p. 68190M (January 2008)

[6] Matschitsch, S., Tschinder, M., Uhl, A.: Comparison of compression algorithms' impact on iris recognition accuracy. In: Lee, S.-W., Li, S.Z. (eds.) ICB 2007. LNCS, vol. 4642, pp. 232-241. Springer, Heidelberg (2007)

[7] Philips, P.J., Bowyer, K.W., Flynn, P.J.: Comments on the CASIA version 1.0 iris data set. IEEE Transactions on Pattern Analysis and Machine Intelligence 29(10), 1869-1870 (2007)

[8] Proenca, H., Alexandre, L.A.: UBIRIS: a noisy iris image database. In: Roli, F., Vitulano, S. (eds.) ICIAP 2005. LNCS, vol. 3617, pp. 970-977. Springer, Heidelberg (2005)

[9] Rakshit, S., Monro, D.M.: Effects of sampling and compression on human iris verification. In: Proceedings of the IEEE International Conference on Acustics, Speech, and Signal Processing (ICASSP 2006), Tolouse, France, pp. II-337-II-340 (2006)

[10] Taubman, D., Marcellin, M.W.: JPEG2000 - Image Compression Fundamentals, Standards and Practice. Kluwer Academic Publishers, Dordrecht (2002)

[11] von Seelen, U.: IrisCode template compression and its effects on authentication performance. In: Biometrics Consortium Conference 2003 (September 2003) 